\title{
On a Cartan Formula for the Algebraic Steenrod Operations Associated with a Pair of Hopf Algebras
}

\author{
By \\ Basil AL-Hashimi* and Frank S. Brenneman*
}

\section{Introduction}

S. Araki [2] and R. Vazquez [7] defined two types of Steenrod squaring operations in the spectral sequence mod 2 associated with a fibre space in the sense of Serre, by using the cubical singular cohomology theory. They computed the Cartan formula and Adams relation. H. Uehara [5] established an algebraic analogy to their work. He discovered and investigated the Steenrod operations in the Adams spectral sequence associated with a pair of Hopf algebras.

In Paragraph 1 of this paper, [5] is modified and reviewed. It is shown that the operations are independent of the higher homotopies under a certain filtration condition and the Cartan formula is obtained.

\section{$\S 1$. Modification of [5]}

In [5], a graded differential algebra with a decreasing filtration and cup-i-products was defined. Theorem 2 of [5] stated that in Adams filtered complex associated with a pair of Hopf algebras over $Z_{2}$, there exist $Z_{2}$-linear maps such that the Adams filtered complex is then a graded differential algebra with a decreasing filtration and cup-i-products. The proof of this theorem is not complete, in fact there is some question as to whether it can be proved using Definition 1 of [5].

Communicated by N. Shimada, April 26, 1974.

* Department of Mathematics, Lehigh University, Bethlehem, Pennsylvania, U.S.A. 
In this chapter Definition 1 of [5] is modified and restated as Definition $\left(^{*}\right)$. With this definition the results of [5] are all true. If the change in definition affects the proof of propositions or theorems of [5], they are proved in this chapter. It should further be noted, if Definition 1 of [5] is satisfied then Definition $\left({ }^{*}\right)$ is satisfied but the converse may not be true.

Definition $\left(^{*}\right)$. By a graded differential algebra $G=\left\{C, \delta, F, \bigcup_{i}\right\}$ with a decreasing filtration $F$ and with cup-i-products $\bigcup_{i}$, we mean 1) a graded cochain complex $C$ over the field $Z_{2}$ :

$$
C: C^{0} \longrightarrow C^{1} \longrightarrow \ldots \rightarrow C^{n} \stackrel{\delta^{n}}{\longrightarrow} C^{n+1} \longrightarrow \ldots,
$$

where $\delta^{n}: C^{n} \rightarrow C^{n+1}$ is a morphism of graded vector spaces over $Z_{2}$,

2) for each integer $p, F^{p} C$ is a subcomplex of $C$ such that

i) $F^{p+1} C$ is a subcomplex of $F^{p} C$ (in notation: $F^{p} C \supset F^{p+1} C$ )

ii) $F^{p} C=C$ if $p \leqq 0$, and

iii) $F^{p} C^{n}=0$ if $p>n$,

3) for each integer $i$ there exists a $Z_{2}$-linear map $\cup: C \otimes C \rightarrow C$ such that if $x \in F^{p} C^{m, s}$ and $y \in F^{q} C^{n, t}$, then $x \bigcup_{i} y \in F^{\alpha} C^{m+n-i, s+t}$ for

$$
\alpha=\left\{\begin{array}{l}
\operatorname{Max}\{p+q-i, p, q\} \text { if } p=q \\
\operatorname{Max}\{p+q-i, \operatorname{Min}\{p, q\}+1\} \text { if } p \neq q,
\end{array}\right.
$$

where $x \bigcup_{i} y=\bigcup_{i}(x \otimes y), x \cup y=x \bigcup_{o} y$ in notations, and $s, t$ stands for gradings. $\bigcup_{i}$ satisfies the following conditions:

i) $\bigcup_{i}$ is trivial if $i<0$,

ii) For $x \in F^{q} C^{m}$ and $y \in F^{p} C^{n}, x \cup_{i} y=0$ if $i>m$ or $n$,

iii) $x \cup(y \cup z)=(x \cup y) \cup z$,

iv) $1 \cup x=x \cup 1$ for some $1 \in C^{0,0}$, and

v) $\delta\left(x \cup_{i} y\right)=x \cup_{i-1} y+y \cup_{i-1} x+\delta x \cup_{i} y+x \bigcup_{i} \delta y$ :

Let $Z_{\gamma}^{p, q}=\left\{x \in F^{p} C^{p+q} \mid \delta x \in F^{p+r} C^{p+q+1}\right\}$

$B_{r}^{p, q}=\left\{x \in F^{p} C^{p+q} \mid\right.$ there exists $y \in F^{p-r} C^{p+q-1}$ with $\left.\delta y=x\right\}$, $Z_{\infty}^{p, q}=\left\{x \in F^{p} C^{p+q} \mid \delta x=0\right\}$, and $B_{\infty}^{p, q}=\left\{x \in F^{p} C^{p+q} \mid\right.$ there exists $y \in C^{p+q-1}$ with $\left.\delta y=x\right\}$. 
Define $\quad E_{r}^{p, q}=\frac{Z_{r}^{p, q}}{Z_{r-1}^{p+1, q-1}+B_{r-1}^{p, q}} \quad \infty \geqq r \geqq 1$

Thus, we have a spectral sequence $\left\{E_{r}, d_{r} \mid r \geqq 1\right\}$, where $d_{r}: E_{r}^{p, q} \rightarrow$ $E_{r}^{p+r, q-r+1}$, is the composition

$$
\begin{aligned}
E_{r}^{p, q}= & \frac{Z_{r}^{p, q}}{Z_{r-1}^{p+1, q-1}+B_{r-1}^{p, q}} \stackrel{\delta}{\longrightarrow} \frac{Z_{r}^{p+r, q-r+1}}{B_{r-1}^{p+r, q-r+1}} \\
& \stackrel{i}{\longrightarrow} \frac{Z_{r}^{p+r, q-r+1}}{Z_{r-1}^{p+r+1, q-r}+B_{r-1}^{p+r, q-r+1}}=E_{r}^{p+r, q-r+1},
\end{aligned}
$$

for more details see [4].

Let us define a map $\theta_{i}: C \rightarrow C$ by $\theta_{i}(x)=x \bigcup_{i} x+x \bigcup_{i+1} \delta x$.

Proposition 1. $\theta_{i}$ induces Steenrod operations ${ }_{B} S t_{i},{ }_{F} S t_{i}$ in the spectral sequence associated with the algebraic system $G$ such that

$$
{ }_{B} S t_{i}: E_{r}^{p, q} \longrightarrow E_{2 \gamma-2}^{2 p-i, 2 q} \text { for } \infty \geqq \gamma \geqq 2,
$$

and

$$
{ }_{F} S t_{i}: E_{r}^{p, q} \longrightarrow E_{r}^{p, 2 q+p-i} \text { for } \infty \geqq \gamma \geqq 1
$$

They are all $Z_{2}$-homomorphisms.

Proof. See [5].

Lemma 1. Define $\tilde{\theta}_{i}: C \rightarrow C$ by $\tilde{\theta}_{i}(x)=x \bigcup_{i} x+\delta x \bigcup_{i+1} x$, then ${ }_{B} S t_{i}$ $={ }_{B} \widetilde{S t_{i}}$ and ${ }_{F} S t_{i}={ }_{F} \widetilde{S t_{i}}$, where ${ }_{B} \widetilde{S t_{i}},{ }_{F} \widetilde{S t_{i}}$ are the Steenrod operations induced by $\tilde{\theta}_{i}$.

Proof. $\theta_{i}(x)+\tilde{\theta}_{i}(x)=x \bigcup_{i} x+x \bigcup_{i+1} \delta x+x \bigcup_{i} x+\delta x \bigcup_{i+1} x=x \bigcup_{i+1} \delta x+\delta x \bigcup_{i+1} x$

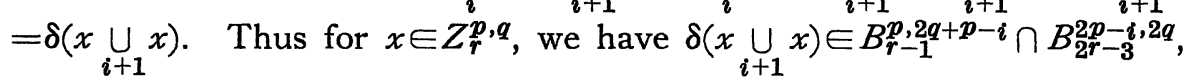
and therefore

$$
{ }_{F} S t_{i}={ }_{F} \widetilde{S t_{i}} \text { and }{ }_{B} S t_{i}={ }_{B} \widetilde{S t_{i}}
$$

To prepare for the existence of a graded differential algebra $G=$ $\left\{C, \delta, F, \bigcup_{i}\right\}$ with a decreasing filtration $F$ and with cup-i-products $\bigcup_{i}$, we need the following proposition. 
Proposition 2. Let $A$ be a cocommutative Hopf algebra over $Z_{2}$ and let $\Delta: A \rightarrow A \otimes A$ be the comultiplication. If $\in: \mathscr{X} \rightarrow Z_{2}$ is a $Z_{2}$-split exact resolution of the $A$-module $Z_{2}$, then there exists a sequence of $\Delta$ homomorphisms $h^{i}: \mathfrak{X} \rightarrow \mathfrak{X} \otimes \mathfrak{X}$ for $i \geqq 0$, such that

1) $h^{0}$ is a grade preserving $\Delta$-chain map,

2) for $i>0 h^{i}$ is a $\Delta$-chain homotopy connecting $h^{i-1}$ with $\rho h^{i-1}$ which raises the homological dimensions by $i$ and preserves the gradings, where $\rho: \mathscr{X} \otimes \mathfrak{X} \rightarrow \mathscr{X} \otimes \mathfrak{X}$ is the twisting chain map. Moreover, if $K^{i}$ for $i \geqq 0$ satisfies 1 ) and 2 ), then there exists a sequence of $\Delta$-maps $S^{i}$ for $i \geqq 0$ such that

3) $S^{0}=0$ and

4) For $i \geqq 0, S^{i+1} \delta+d S^{i+1}=h^{i}+k^{i}+S^{i}+\rho S^{i}$, where $d, \delta$ are boundary operators for $\mathfrak{X} \otimes \mathfrak{X}$ and $\mathscr{X}$ respectively.

Proof. See [5] and [6].

Consider the diagram

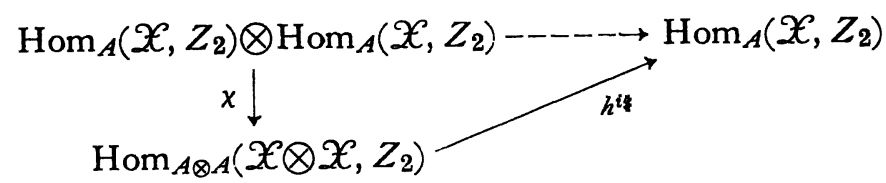

where $\chi$ is the $Z_{2}$-chain map defined by

$$
\chi(f \otimes g)(x \otimes y)=f(x) g(y) \text { for } f, g \in \operatorname{Hom}_{A}\left(\mathfrak{X}, Z_{2}\right)
$$

and for $x, y \in \mathfrak{X}$.

Definition. The cup-i-product $\bigcup_{i}$ in the cochain complex $C=$ $\operatorname{Hom}_{A}\left(\mathscr{X}, Z_{2}\right)$ is denoted by $h^{i *} \chi$.

Denoting $\operatorname{Hom}_{A}^{s}\left(X_{p}, Z_{2}\right)$ by $C^{p, s}$ for each homological dimension $p \geqq 0$ and the grading $s \geqq 0$, we have the cochain complex

$$
C^{* s}=\left\{C^{p, s} \text { for } p=0,1, \ldots, n, \ldots\right\}
$$

such that $C=\left\{C^{* s} \mid s=0,1, \ldots\right\}$. Then

$$
f \bigcup_{i} g=\bigcup_{i}(f \otimes g) \in C^{p+q-i, s+t}
$$


for $f \in C^{p, s}$ and $g \in C^{q, t}$. By the definition of $\bigcup_{i}$, we have,

Lemma 2. $\delta\left(f \cup_{i} g\right)=f \bigcup_{i-1} g+g \cup_{i-1} f+\delta f \bigcup_{i} g+f \bigcup_{i} \delta g$.

J. F. Adams [1] and A. Zachariou [8] computed explicitly a $\Delta$ homomorphism $h^{i}$ in case when $\mathcal{X}$ is the bar resolution $B(A)$. If $\Delta(a)$ $=\sum a^{\prime} \otimes a^{\prime \prime}$ for $a \in A$, then we have

$$
\begin{aligned}
h_{n}^{o}\left(\left[a_{1}\left|a_{2}\right| \ldots \mid a_{n}\right]\right)= & 1 \otimes\left[a_{1}|\ldots| a_{n}\right] \\
& +\sum_{1 \leqq \rho \leqq n}\left[a_{1}^{\prime}|\ldots| a_{\rho}^{\prime}\right] \otimes a_{1}^{\prime \prime} \ldots a_{\rho}^{\prime \prime}\left[a_{\rho+1}|\ldots| a_{n}\right]
\end{aligned}
$$

for odd $i$,

$$
\begin{aligned}
h_{n}^{i}\left(\left[a_{1} \mid \ldots a_{n}\right]\right) & =\sum_{0 \leqq \rho_{0}<\rho_{1}<\ldots<\rho_{i} \leq n}\left[a_{1}^{\prime}|\ldots| a_{\rho_{0}}^{\prime}\left|a_{\rho_{0}+1}^{\prime} \ldots a_{\rho_{1}}^{\prime}\right| a_{\rho_{1}+1}^{\prime}|\ldots| a_{\rho_{2}}^{\prime} \mid \ldots\right. \\
& \left.\ldots\left|a_{\rho_{i-1}+1}^{\prime} \ldots a_{\rho_{i}}^{\prime}\right| a_{\rho_{i+1}}|\ldots| a_{n}\right] \otimes a_{1}^{\prime \prime} \ldots a_{\rho_{0}[}^{\prime \prime}\left[a_{\rho_{0}+1}^{\prime \prime} \mid \ldots\right. \\
& \left.\ldots\left|a_{\rho_{1}}^{\prime \prime} a_{\rho_{1}+1}^{\prime \prime} \ldots a_{\rho_{2}}^{\prime \prime}\right| \ldots\left|a_{\rho_{i-1}+1}^{\prime \prime}\right| \ldots \mid a_{\rho_{i}}^{\prime \prime}\right]
\end{aligned}
$$

for even $i$,

$$
\begin{aligned}
h_{n}^{i}\left(\left[a_{1}|\ldots| a_{n}\right]\right) & =\sum_{0 \leqq \rho_{0}<\rho_{1}<\ldots<\rho_{i} \leqq n}\left[a_{1}^{\prime}|\ldots| a_{\rho_{0}}^{\prime}\left|a_{\rho_{0}+1}^{\prime} \ldots a_{\rho_{1}}^{\prime}\right| \ldots\left|a_{\rho_{i-1}+1}^{\prime}\right| \ldots \mid a_{\rho_{i}}^{\prime}\right] \\
& \left.\bigotimes a_{1}^{\prime \prime} \ldots a_{\rho_{0}\left[a_{\rho_{0}+1}^{\prime \prime}\right.}^{\prime \prime}|\ldots| a_{\rho_{1}}^{\prime \prime}|\ldots| a_{\rho_{i-1}+1}^{\prime \prime} \ldots a_{\rho_{i+1}}^{\prime \prime}|\ldots| a_{n}\right] .
\end{aligned}
$$

The above $h^{i}$ was computed in the following way. Let $s$ be the contracting homotopy for $B(A)$, then $t=s \otimes 1+\sigma \varepsilon \otimes s$ is a contracting homotopy for $B(A) \otimes B(A)$. Define $h^{i}$ by the following inductive formulas

1) $h_{o}^{o}=\Delta$

2) $h_{n-1}^{o} s_{n-1} t_{n-1}^{o} \quad$ for $n>0$

3) $h_{o}^{j}=t_{j-i}\left(h_{o}^{j-1}+\rho h_{o}^{j-1}\right) \quad$ for $j \geqq 1$

4) $h_{n}^{j} s_{n-1}=t_{n+j-1}\left(h_{n}^{j-1}+\rho h_{n}^{j-1}\right) s_{n-1}+t_{n+j-1} h_{n-1}^{j}$ for $n>0$ and $j \geqq 1$

5) $h_{n}^{j}(a x)=\Delta a h_{n}^{j}(x)$ for $j \geqq 0, a \in A, x \in I(A)^{n}$ and $n \geqq 1$.

Remark 1. $h_{o}^{j}=0$ for $j>0$.

Remark 2. $h_{q}^{p}=0$ for $p>q$. 
Remark 3. $h_{o}^{j} s_{n-1}=t_{n+j-1} h_{n}^{j-1} s_{n-1}+t_{n+j-1} h_{n-1}^{j}$ for $j \geqq 1$ and $n \geqq 1$.

Let $(\Gamma, \Lambda)$ be a pair of connected locally finite cocommutative Hopf algebras over $Z_{\mathbf{2}}$ such that, the subhopf algebra $\Lambda$ is central in $\Gamma$, in the sense that

$$
a b=b a \quad \text { if } \quad a \in \Lambda, b \in \Gamma .
$$

We are going to associate with the pair $(\Gamma, \Lambda)$ a graded differential algebra $G(\Gamma, \Lambda)=\left\{C, \delta, F, \bigcup_{i}\right\}$ with a decreasing filtration $F$ and with cup-i-products $\underset{i}{\cup}$.

J. F. Adams [1] introduced a filtration in the bar construction $B(\Gamma)$, in the following way. For each integer $p$ define a subcomplex $F_{p} B(\Gamma)$ of $B(\Gamma)$ such that $F_{p} B(\Gamma)_{n}$ is the $\Gamma$-submodule of $B(\Gamma)_{n}=\Gamma \otimes I(\Gamma)^{n}$ generated by elements of the form $\gamma\left[\gamma_{1}|\ldots| \gamma_{n}\right]$ with the property $\gamma_{s} \in I(\Lambda)$ for at least $(n-p)$ values of $s$. Then it is immedeidate to see that $F$ is the canonical increasing filtration in $B(\Gamma)$.

Define the product filtration $F^{*}$ in $B(\Gamma) \otimes B(\Gamma)$ by

$$
\stackrel{*}{F}_{p}(B(\Gamma) \otimes B(\Gamma))=\bigcup_{p \geq s \geq 0} F_{p-s} B(\Gamma) \otimes F_{s} B(\Gamma) .
$$

Then $(B(\Gamma) \otimes B(\Gamma), \stackrel{*}{F})$ is a resolution of the $\Gamma \otimes \Gamma$-module $Z_{2}$ with the increasing filtration $\stackrel{*}{F}$. Let $\Delta: \Gamma \rightarrow \Gamma \otimes \Gamma$ be the cocommutative diagonal and let $\rho$ be the twisting chain map of $B(\Gamma) \otimes B(\Gamma)$. Then we have

Proposition 3. There exists a sequence of $\Delta$-homomorphisms $h^{i}$ : $B(\Gamma) \rightarrow B(\Gamma) \otimes B(\Gamma)$ for $i \geqq 0$ such that

1) $h^{0}$ is a $\Delta$-chain map which preserves grading and filtration,

2) $h^{i}$ is a $\Delta$-chain homotopy connecting $h^{i-1}$ and $\rho h^{i-1}$ which preserves grading, raises homological dimension by $i$, and satisfies the filtration condition

$$
\begin{aligned}
& h^{i}\left(F_{p} B(\Gamma)\right) \subset \stackrel{*}{F}_{\alpha}(B(\Gamma) \otimes B(\Gamma)) \\
& \text { for } \quad \alpha=\operatorname{Min}\{2 p, p+i\} .
\end{aligned}
$$

Proof. The particular $h_{n}^{i}$ defined previously satisfies the Proposition, [5]. 
Let $(C, \delta)$ be the cochain complex $\operatorname{Hom}_{\Gamma}\left(B(\Gamma), Z_{2}\right)$ over $Z_{2}$. For each integer $p$ define a subcomplex $F^{p}(C)$ by the image of

$$
\operatorname{Hom}_{\Gamma}\left(\frac{B(\Gamma)}{F_{p-1} B(\Gamma)}, Z_{2}\right)
$$

under the dual of the projection

$$
\pi: B(\Gamma) \longrightarrow \frac{B(\Gamma)}{F_{p-1} B(\Gamma)}
$$

Then $(C, \delta, F)$ is a cochain complex with a decreasing filtration. Let us call it Adams filtered complex associated with $(\Gamma, \Lambda)$.

Proposition 4. Let $(C, \delta, F)$ be the Adams filtered complex associated with a pair of Hopf algebras $(\Gamma, \Lambda)$ over $Z_{2}$. Then there exist $Z_{2}$-linear maps $\bigcup_{i}: C \otimes C \rightarrow C$ such that $G(\Gamma, \Lambda)=\left\{C, \delta, F, \bigcup_{i}\right\}$ is a graded differential algebra with a decreasing filtration $F$ and cup-i-products $\bigcup_{i}$, in the sense of Definition (*).

Proof. Let $h^{i}: B(\Gamma) \rightarrow B(\Gamma) \otimes B(\Gamma)$ be the $\Delta$-homomorphism in Proposition 3 and define $\bigcup_{i}: C \otimes C \rightarrow C$ by $h^{i k} \chi$. Since $\bigcup_{i}$ is the cup-iproduct in $C=\operatorname{Hom}_{\Gamma}\left(B(\stackrel{i}{i}), Z_{2}\right)$, it is easy to see that $\bigcup_{i}^{i}$ satisfies all the necessary condition except the filtration condition. Consequently, it is sufficient to show that if $f \in F^{p} C^{m, s}$ and $g \in F^{q} C^{n, t}$, then $f \cup_{i} g \in F^{\alpha} C^{m+n-i, s+t}$ for

$$
a=\left\{\begin{array}{l}
\operatorname{Max}\{p+q-i, p, q\} \text { if } p=q \\
\operatorname{Max}\{p+q-i, \operatorname{Min}\{p, q\}+1\} \text { if } p \neq q
\end{array} .\right.
$$

Consider first the case when $\alpha=p+q-i$. By Proposition 3 $h^{i}\left(F_{\alpha-1} B(\Gamma)\right) \subset \stackrel{*}{F_{2(\alpha-1)}}(B(\Gamma) \otimes B(\Gamma)) \cap \stackrel{*}{F}_{(\alpha-1)+i}(B(\Gamma) \otimes B(\Gamma))$

thus

$$
\begin{aligned}
h^{i}\left(F_{\alpha-1} B(\Gamma)\right) & \subset F_{(\alpha-1)+i}^{*}(B(\Gamma) \otimes B(\Gamma)) \\
& =F_{p+q-1}^{*}(B(\Gamma) \otimes B(\Gamma)) .
\end{aligned}
$$

Then 


$$
\begin{aligned}
\left(f \cup_{i} g\right)\left(F_{\alpha-1} B(\Gamma)\right) & \subset(f \otimes g){ }^{*}{ }_{p+q-1}(B(\Gamma) \otimes B(\Gamma)) \\
& =(f \otimes g) \sum F_{\xi} B(\Gamma) \otimes F_{\sigma} B(\Gamma)
\end{aligned}
$$

where $\xi+\sigma=p+q-1$ and thus

$$
\left(f \cup_{i} g\right)\left(F_{\alpha-1} B(\Gamma)\right)=0
$$

because

$$
\xi<p \text { or } \sigma<q
$$

If $\alpha=p$, then $p=q$. In this case also

$$
\begin{aligned}
\left(f \bigcup_{i} g\right)\left(F_{p-1} B(\Gamma)\right) & \subset(f \otimes g) \stackrel{*}{F}_{2 p-2}(B(\Gamma) \otimes B(\Gamma) \\
& =(f \otimes g) \stackrel{*}{F}_{p+q-2}(B(\Gamma) \otimes B(\Gamma)) \\
& =0 .
\end{aligned}
$$

If $\alpha=p+1$, then $p \leqq q-1$ and

$$
\begin{aligned}
\left(f \cup_{i} g\right)\left(F_{p} B(\Gamma)\right) & \subset(f \otimes g) \stackrel{*}{F}_{2 p}(B(\Gamma) \otimes B(\Gamma)) \\
& \subset(f \otimes g) F_{p+q-1}(B(\Gamma) \otimes B(\Gamma)) \\
& =0 .
\end{aligned}
$$

Similarly when $a=q+1$. Hence, the proof is completed.

From Propositions 4 and 1 we obtain,

Proposition 5. Let $(\Gamma, \Lambda)$ be a pair of connected locally finite cocommutative Hopf algebras over $Z_{2}$ such that $\Lambda$ is central in $\Gamma$, and let $\left\{E_{r}, d_{r}\right\}$ be the Adams spectral sequence associated with the system $G(\Gamma, \Lambda)$. Then there exist algebraic Steenrod operations

$$
{ }_{B} S t_{i}: E_{\gamma}^{p, q} \longrightarrow E_{2 \gamma-2}^{2 p-i, 2 q} \quad \text { for } \quad \infty \geqq \gamma \geqq 2
$$

and

$$
{ }_{F} S t_{i}: E_{r}^{p, q} \longrightarrow E_{r}^{p, 2 q+p-i} \text { for } \infty \geqq \gamma \geqq 1
$$

Proposition 6. Let $(\Gamma, \Lambda)$ and $\left(\Gamma^{\prime}, \Lambda^{\prime}\right)$ be pairs of Hopf algebras over $Z_{2}$ both of which satisfy the conditions stated before, and let $E_{r}$ and $E_{\gamma}^{\prime}$ be the Adams spectral sequence associated with $G(\Gamma, \Lambda)$ and $G\left(\Gamma^{\prime}, \Lambda^{\prime}\right)$ respectively. If $f:(\Gamma, \Lambda) \rightarrow\left(\Gamma^{\prime}, \Lambda^{\prime}\right)$ is a morphism of pairs of Hopf alge- 
bras, then $f$ induces a sequence of homomorphisms

$$
\varphi_{r}: E_{\gamma}^{\prime} \longrightarrow E_{r} \text { for } \gamma \geqq 1
$$

such that

$$
\varphi_{r} S t_{i}={ }_{F} S t_{i} \varphi_{r}
$$

and

$$
\varphi_{2 \gamma-2} S t_{i}={ }_{B} S t_{i} \varphi_{\gamma} \text { for } \gamma \geqq 2 .
$$

Proof. See [5].

Proposition 7. Let $k^{i}: B(\Gamma) \rightarrow B(\Gamma) \otimes B(\Gamma)$ for $i=0,1, \ldots, n, \ldots$, be a sequence of $\Delta$-homomorphisms such that

1) $k^{0}$ is a $\Delta$-chain map which preserves grading and filtration.

2) $k^{i}$ is a $\Delta$-chain homotopy connecting $k^{i-1}$ and $p k^{i-1}$ which preserves gradings, raises homological dimension by $i$, and satisfies the filtration condition

$$
k^{i}\left(F_{p} B(\Gamma)\right) \subset \stackrel{*}{F}_{\alpha}(B(\Gamma) \otimes B(\Gamma)) \quad \text { for } \quad a=\operatorname{Min}\{2 p, p+i\} .
$$

Then there exists a $\Delta$-homomorphism $E^{i}: B(\Gamma) \rightarrow B(\Gamma) \otimes B(\Gamma)$ such that

i) $E^{0}=0$

ii) $h^{i}+k^{i}=E^{i}+\rho E^{i}+D E^{i+1}+E^{i+1} d$.

Moreover if $E^{i}\left(F_{p} B(\Gamma) \subset \stackrel{*}{F}_{\alpha}(B(\Gamma) \otimes B(\Gamma))\right.$ for $\alpha=\operatorname{Min}\{2 p, p+i\}$ then the Steenrod operations ${ }_{B} s_{i},{ }_{F} s_{i}$ induced by $k^{i}$, are equal to ${ }_{B} S t_{i},{ }_{F} S t_{i}$ the Steenrod operations induced by $h^{i}$ respectively.

Proof. The first part is Proposition 2. For the rest of the proof let $\bar{i}=k^{i} \chi$, and define

$$
\psi_{i}: \operatorname{Hom}_{\Gamma}\left(B(\Gamma), Z_{2}\right) \longrightarrow \operatorname{Hom}_{\Gamma}\left(B(\Gamma), Z_{2}\right)
$$

by $\psi_{i}(\dot{\xi})=\xi \bigcup_{i} \xi+\xi \bigcup_{i+1} \delta \xi$, then $\psi_{i}$ induces

$$
{ }_{B} t_{i}: E_{\tilde{\gamma}}^{p, q} \longrightarrow E_{2 \gamma-2}^{2 p-i, 2 q} \text { for } \infty \geqq \gamma \geqq 2
$$

and

$$
{ }_{F} s t_{i}: E_{\gamma}^{p, q} \longrightarrow E_{\gamma}^{p, 2 q+p-i} \text { for } \quad \infty \geqq \gamma \geqq 1
$$


Now $\theta_{i}(\xi)+\psi_{i}(\xi)=\xi \cup_{i} \xi+\xi \cup_{i+1} \delta \xi+\xi \bigcup_{i} \xi+\xi \bigcup_{i+1} \delta \xi$ and thus by the first part we can show that

$$
\begin{aligned}
\theta_{i}(\xi)+\psi_{i}(\xi)= & D^{\sharp}\left(E^{(i+1) *} \chi(\xi \otimes \xi)+E^{(i+2) *} \chi(\xi \otimes \delta \xi)\right) \\
& +E^{(i+2) *} \chi(\delta \xi \otimes \delta \xi)
\end{aligned}
$$

Since $E^{i} F_{p} \subset F_{\alpha}$ for $a=\operatorname{Min}\{2 p, p+i\}$, thus by Proposition 4 we have, for $\xi_{1} \in F^{p} \operatorname{Hom}_{\Gamma}\left(B(\Gamma), Z_{2}\right)$ and $\xi_{2} \in F^{q} \operatorname{Hom}_{\Gamma}\left(B(\Gamma), Z_{2}\right)$,

$$
E^{j k} \chi\left(\xi_{1} \otimes \xi_{2}\right) \in F^{r} \operatorname{Hom}_{\Gamma}\left(B(\Gamma), Z_{2}\right)
$$

where

$$
\gamma=\left\{\begin{array}{lll}
\operatorname{Max}(p+q-j, p, q) & \text { if } & p=q \\
\operatorname{Max}(p+q-j, \operatorname{Min}(p, q)+1) & \text { if } & p \neq q
\end{array}\right.
$$

Therefore one can show that

$$
\begin{gathered}
\theta_{i}(\xi)+\psi_{i}(\xi) \in Z_{\tilde{\tau}-1}^{p+1,2 q+p-i-1} \cap Z_{2 \gamma-3}^{2 p-i+1,2 q-1} \\
+B_{\bar{\tau}-1}^{p, 2 q+p-i} \cap B_{2 \gamma-3}^{2 p-i, 2 q}
\end{gathered}
$$

which means that

$$
{ }_{B} S t_{i}={ }_{B} s t_{i} \text { and }{ }_{F} S t_{i}={ }_{F} S t_{i} .
$$

\section{§ 2. Cartan Formula}

Let $\Gamma$ be a connected, locally finite, cocommutative Hopf algebra over the field $Z_{2}$ and let $\Lambda$ be a Hopf subalgebra of $\Gamma$ which is central in $\Gamma$.

Remark 4. $\Lambda \otimes \Lambda$ is central in $\Gamma \otimes \Gamma$ and

$$
\Delta^{*}=(1 \otimes t \otimes 1)(\Delta \otimes \Delta): \Gamma \otimes \Gamma \longrightarrow \Gamma \otimes \Gamma \otimes \Gamma \otimes \Gamma
$$

is commutative where $t(x \otimes y)=y \otimes x$. Therefore $(\Gamma \otimes \Gamma, \Lambda \otimes \Lambda)$ is a pair of $Z_{2}$-Hopf algebras with the properties of paragraph 1 .

Consider the following diagram 
$\ldots \longrightarrow B(\Gamma \otimes \Gamma)_{n} \longrightarrow \ldots \longrightarrow B(\Gamma \otimes \Gamma)_{1} \stackrel{d}{\longrightarrow} \Gamma \otimes \Gamma \quad B(\Gamma \otimes \Gamma)$

$f_{n} \uparrow \downarrow g_{n}$

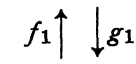

1

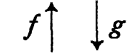

$\ldots \longrightarrow(B(\Gamma) \otimes B(\Gamma))_{n} \longrightarrow \ldots(B(\Gamma) \otimes B(\Gamma))_{1} \stackrel{d^{*}}{\longrightarrow} \Gamma \otimes \Gamma \quad B(\Gamma) \otimes B(\Gamma)$

V.K.A.M. Gugenheim [3], defines $f$ and $g$ as $\Gamma \otimes \Gamma$-chain maps by

$$
\begin{aligned}
& g\left[a_{1} \otimes b_{1}|\ldots| a_{n} \otimes b_{n}\right] \\
& \quad=\sum_{0 \leq \rho \leq n} \varepsilon \sigma\left(a_{\rho+1}\right) \ldots \varepsilon \sigma\left(a_{n}\right)\left[a_{1}|\ldots| a_{\rho}\right] \otimes b_{1} \ldots b_{\rho}\left[b_{\rho+1}|\ldots| b_{n}\right]
\end{aligned}
$$

and

$$
\begin{aligned}
& f\left\{\left[a_{1}|\ldots| a_{p}\right] \otimes\left[b_{1}|\ldots| b_{q}\right]\right\} \\
& \quad=\Sigma\left[c_{1}|\ldots| c_{p+q}\right]
\end{aligned}
$$

where $c_{1}, \ldots, c_{p+q}$ is a shuffle of $a_{1} \otimes 1, \ldots, a_{p} \otimes 1,1 \otimes b_{1}, \ldots, 1 \otimes b_{q}$. Moreover he shows that $g f=I$. Define $H^{i}: B(\Gamma \otimes \Gamma) \rightarrow B(\Gamma \otimes \Gamma) \otimes B(\Gamma \otimes \Gamma)$ by

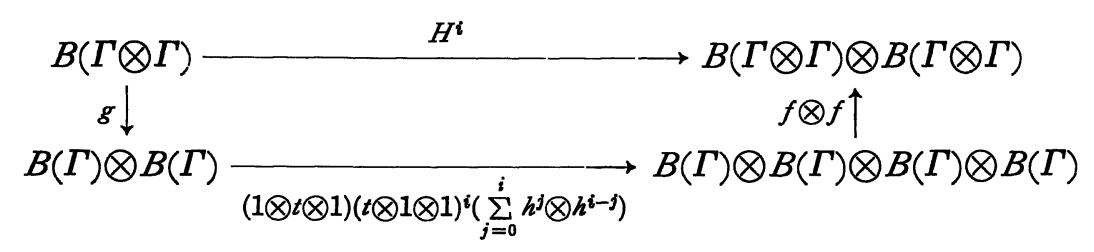

Lemma 3. $H^{i}$ is a $\Delta^{*}$-homomorphism.

Proof. Construct the appropriate diagram and verify commutativity.

\section{Lemma 4.}

$$
H^{i} d+D H^{i}=H^{i-1}+\rho H^{i-1}
$$

Proof.

$$
\begin{aligned}
H^{i} d= & (f \otimes f)(1 \otimes t \otimes 1)(t \otimes 1 \otimes 1)^{i}\left(\sum_{j=0}^{i} h^{j} \otimes h^{i-j}\right) g d \\
= & (f \otimes f)(1 \otimes t \otimes 1)(t \otimes 1 \otimes 1)^{i}\left(\sum_{j=0}^{i} h^{j} \otimes h^{i-j}\right) d^{*} g \\
= & (f \otimes f)(1 \otimes t \otimes 1)(t \otimes 1 \otimes 1)^{i}\left[h^{0} \otimes h^{i}+h^{i} \otimes h^{0}+\sum_{j=1}^{i-1} h^{j} \otimes h^{i-j}\right] d^{*} g \\
= & (f \otimes f)(1 \otimes t \otimes 1)(t \otimes 1 \otimes 1)^{i}\left[h^{0} d \otimes h^{i}+h^{0} \otimes h^{i} d+h^{i} d \otimes h^{0}+h^{i} \otimes h^{0} d\right. \\
& \left.+\sum_{j=1}^{i-1} h^{j} d \otimes h^{i-j}+\sum_{j=1}^{i-1} h^{j} \otimes h^{i-j} d\right] g \\
= & (f \otimes f)(1 \otimes t \otimes 1)(t \otimes 1 \otimes 1)^{i}\left[\delta h^{0} \otimes h^{i}+h^{0} \otimes \delta h^{i}+h^{0} \otimes h^{i-1}\right.
\end{aligned}
$$


$+h^{0} \otimes \rho h^{i-1}+\delta h^{i} \otimes h^{0}+h^{i-1} \otimes h^{0}+\rho h^{i-1} \otimes h^{0}+h^{i} \otimes \delta h^{0}$

$\left.+\sum_{j=1}^{i-1}\left(\delta h^{j}+h^{j-1}+\rho h^{j-1}\right) \otimes h^{i-j}+\sum_{j=1}^{i-1} h^{j} \otimes\left(\delta h^{i-j}+h^{i-j-1}+\rho h^{i-j-1}\right)\right] g$

$=(f \otimes f)(1 \otimes t \otimes 1)(t \otimes 1 \otimes 1)^{i}(\delta \otimes 1+1 \otimes \delta)\left(\sum_{j=0}^{i} h^{j} \otimes h^{i-j}\right) g$

$+(f \otimes f)(1 \otimes t \otimes 1)(t \otimes 1 \otimes 1)^{i}(t \otimes 1 \otimes 1+1 \otimes 1 \otimes t)\left(\sum_{j=0}^{i-1} h^{j} \otimes h^{i-j-1}\right) g$

$=D H^{i}+(f \otimes f)(1 \otimes t \otimes 1)(t \otimes 1 \otimes 1)^{i-1}\left(\sum_{j=0}^{i-1} h^{j} \otimes h^{i-j-1}\right) g$

$+(f \otimes f)(1 \otimes t \otimes 1)(t \otimes 1 \otimes 1)^{i}(1 \otimes 1 \otimes t)\left(\sum_{j=0}^{i-1} h^{j} \otimes h^{i-j-1}\right) g$

$=D H^{i}+H^{i-1}+(f \otimes f)(1 \otimes t \otimes 1)(t \otimes 1 \otimes 1)^{i}(1 \otimes 1 \otimes t)\left(\sum_{j=0}^{i-1} h^{j} \otimes h^{i-j-1}\right) g$,

We only need to show that

$(f \otimes f)(1 \otimes t \otimes 1)(t \otimes 1 \otimes 1)^{i}(1 \otimes 1 \otimes t)\left(\sum_{j=0}^{i-1} h^{j} \otimes h^{i-j-1}\right) g=\rho H^{i-1}$,

Case 1. $i$ is even, then

$(1 \otimes t \otimes 1)(t \otimes 1 \otimes 1)^{i}(1 \otimes 1 \otimes t)=(1 \otimes t \otimes 1)(1 \otimes 1 \otimes t)$

$=\rho(1 \otimes t \otimes 1)(t \otimes 1 \otimes 1)=\rho(1 \otimes t \otimes 1)(t \otimes 1 \otimes 1)^{i-1}$

Case 2. $i$ is odd, then

$(1 \otimes t \otimes 1)(t \otimes 1 \otimes 1)^{i}(1 \otimes 1 \otimes t)=(1 \otimes t \otimes 1)(t \otimes 1 \otimes 1)(1 \otimes 1 \otimes t)$

$=\rho(1 \otimes t \otimes 1)(t \otimes 1 \otimes 1)^{i-1}$

Thus

$(f \otimes f)(1 \otimes t \otimes 1)(t \otimes 1 \otimes 1)^{i}(1 \otimes 1 \otimes t)\left(\sum_{j=0}^{i-1} h^{j} \otimes h^{i-j-1}\right) g$

$=(f \otimes f) \rho(1 \otimes t \otimes 1)(t \otimes 1 \otimes 1)^{i-1}\left(\sum_{j=0}^{i-1} h^{j} \otimes h^{i-j-1}\right) g$

$=\rho(f \otimes f)(1 \otimes t \otimes 1)(t \otimes 1 \otimes 1)^{i-1}\left(\sum_{j=0}^{i-1} h^{j} \otimes h^{i-j-1}\right) g=\rho H^{i-1}$.

This completes the proof.

\section{Lemma 5.}

$H^{i} F_{p}^{\prime} B(\Gamma \otimes \Gamma) \subset{\stackrel{*}{F_{\alpha}^{\prime}}}_{\alpha}(B(\Gamma \otimes \Gamma) \otimes B(\Gamma \otimes \Gamma))$ for $\alpha=\operatorname{Min}\{2 p, p+i\}$.

Proof. It can be shown that $g$ and $f$ preserve the filtration.

Finally we need to show that

$\left(h^{j} \otimes h^{i-j}\right)\left(F_{s} B(\Gamma) \otimes F_{p-s} B(\Gamma)\right) \subset \stackrel{* *}{F}_{\gamma}(B(\Gamma) \otimes B(\Gamma) \otimes B(\Gamma) \otimes B(\Gamma))$

where $\gamma=\operatorname{Min}\{2 p, p+i\}$. 
Note that,

1) $h^{j} F_{s} \subset \stackrel{*}{F}_{\alpha}$ where $\alpha=\operatorname{Min}\{2 s, s+j\}$ and

2) $h^{i-j} F_{p-s} \subset \stackrel{F}{F}_{\beta}$ where $\beta=\operatorname{Min}\{2(p-s), p-s+i-j\}$

Now $\left(h^{j} \otimes h^{i-j}\right)\left(F_{s} \otimes F_{p-s}\right) \subset \stackrel{*}{F}_{\alpha} \otimes \stackrel{*}{F}_{\beta} \in \stackrel{* *}{F}_{\alpha+\beta}$, so we have to show that $a+\beta<\gamma$.

Case 1. $\alpha=2 s, \beta=2(p-s)$ then $\alpha+\beta=2 p$ and we need to show that $i \geqq p$.

$$
p-s+i-j \geqq 2(p-2) \Rightarrow i \geqq p+j-s,
$$

also

$$
a=2 s \Rightarrow j \geqq s \Rightarrow j-s \geqq 0,
$$

thus $i \geqq p$.

Case 2. $\alpha=s+j, \beta=p-s+i-j$ then $\alpha+\beta=p+i$ and we need to show that $i \leqq p$. But $\beta=p-s+i-j \Rightarrow p-s+i-j \leqq 2 p-s \Rightarrow i-j \leqq p-s \Rightarrow$ $i \leqq p-s+j$, also $a=s+j \Rightarrow s+j \leqq 2 s \Rightarrow j \leqq s$, thus $i \leqq p$.

Case 3. $\alpha=s+j, \beta=2 p-2 s$, then $\alpha+\beta=2 p-s+j$. But $s+j \leqq 2 s$ $\Rightarrow j \leqq s \Rightarrow j-s \leqq 0 \Rightarrow \alpha+\beta \leqq 2 p$ also $2 p-2 s \leqq p-s+i-j \Rightarrow p+s \leqq i-j \Rightarrow$ $\alpha+\beta \leqq p+i$.

Therefore $\alpha+\beta \leqq \operatorname{Min}\{2 p, p+i\}=\gamma$

Case 4. $\alpha=2 s, \beta=p-s+i-j$ then $\alpha+\beta=p+s+i-j$.

But $s+j \geqq 2 s \Rightarrow j \geqq s \Rightarrow-j \leqq-s \Rightarrow a+\beta \leqq p+i$, also $p-s+i-j \leqq$ $2 p-2 s \Rightarrow i-j \leqq p-2 \Rightarrow \alpha+\beta \leqq 2 p$, and thus $\alpha+\beta \leqq \operatorname{Min}\{2 p, p+i\}=\gamma$.

Consider a diagram

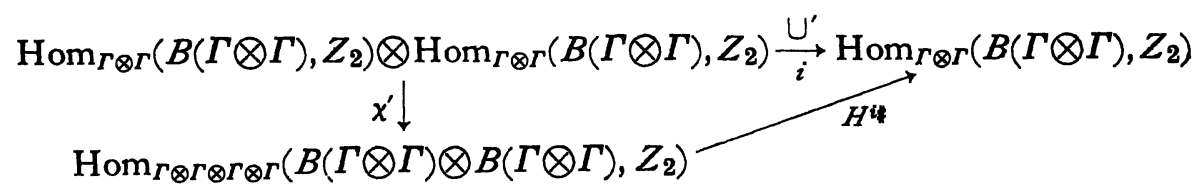

where $\chi^{\prime}(f \otimes g)(x \otimes y)=f(x) g(y)$, then the cup-i-product $\bigcup_{i}^{\prime}$ in the cochain complex $C^{\prime}=\operatorname{Hom}_{\Gamma \otimes \Gamma}\left(B(\Gamma \otimes \Gamma), Z_{2}\right)$ is defined by $H^{i *} \chi^{\prime}$.

Lemma 6. Let $u \in C^{\prime m}$ and $v \in C^{\prime n}$, then $u \bigcup_{i}^{\prime} v=0$ if $i>m$ or $i>n$. 
Proof. $\quad u \cup^{\prime} v=H^{i *} \chi^{\prime}(u \otimes v)$

$$
\begin{aligned}
& =g^{\ddagger}\left(\sum h^{j \sharp} \otimes h^{i-j \ddagger}\right)\left(t^{\sharp} \otimes 1 \otimes 1\right)^{i}\left(1 \otimes t^{\sharp} \otimes 1\right)(f \otimes f)^{*} \chi^{\prime}(u \otimes v) \\
& =g^{\sharp}\left(\sum h^{j \ddagger} \otimes h^{i-j \ddagger}\right)(\chi \otimes \chi)\left(t^{\ddagger} \otimes 1 \otimes 1\right)^{i}\left(1 \otimes t^{\sharp} \otimes 1\right)\left(\xi_{1} \otimes \xi_{2} \otimes \xi_{3} \otimes \xi_{4}\right)
\end{aligned}
$$

where

$$
\begin{gathered}
f(u)=\chi\left(\xi_{1} \otimes \xi_{2}\right) \quad \text { and } f(v)=\chi\left(\xi_{3} \otimes \xi_{4}\right), \\
\xi_{i} \in \operatorname{Hom}_{\Gamma}\left(B(\Gamma), Z_{2}\right) \quad \text { with } \quad\left|\xi_{1}+\xi_{2}\right|=m \quad \text { and }\left|\xi_{3}+\xi_{4}\right|=n . \\
=g^{*}\left(\sum h^{\prime} \otimes h^{i-j *}\right)(\chi \otimes \chi)\left(t^{*} \otimes 1 \otimes 1\right)^{i}\left(\xi_{1} \otimes \xi_{3} \otimes \xi_{2} \otimes \xi_{4}\right)=0
\end{gathered}
$$

if any of the following hold

1) $j>\left|\xi_{1}\right|$

2) $j>\left|\xi_{3}\right|$

3) $i-j>\left|\xi_{2}\right|$

4) $i-j>\left|\xi_{4}\right|$

Now $i>m=\left|\xi_{1}\right|+\left|\xi_{2}\right| \Rightarrow j+i-j>\left|\xi_{1}\right|+\left|\xi_{2}\right| \Rightarrow$ either $j>\left|\xi_{1}\right|$ or $i-j>\left|\xi_{2}\right|$ $\Rightarrow u \cup_{i}^{\prime} v=0$ also $i>n=\left|\xi_{3}\right|+\left|\xi_{4}\right| \Rightarrow$ either $j>\left|\xi_{3}\right|$ or $i-j>\left|\xi_{4}\right| \Rightarrow u \bigcup_{i}^{\prime} v=0$.

Lemma 7. $\delta^{\prime}\left(x \bigcup_{i}^{\prime} y\right)=x \cup_{i-1}^{\cup^{\prime}} y+y \underset{i-1}{\cup^{\prime}} x+\delta^{\prime} x \bigcup_{i}^{\cup^{\prime}} y+x \bigcup_{i}^{\bigcup^{\prime}} \delta^{\prime} y$.

Proof. Straightforward, since

$$
H^{i} d+D H^{i}=H^{i-1}+\rho H^{i-1} .
$$

Let $\left(C^{\prime}, \delta^{\prime}, F^{\prime}\right)$ be the Adams filtered complex associated with $(\Gamma \otimes \Gamma$, $\Lambda \otimes \Lambda)$, then by Lemmas $3,4,5,6$ and Proposition 3 , we have that $G(\Gamma \otimes \Gamma, \Lambda \otimes \Lambda)=\left\{C^{\prime}, \delta^{\prime}, F^{\prime}, \bigcup_{i}^{\prime}\right\}$ is a graded differential algebra with a decreasing filtration $F^{\prime}$ and with cup-i-products $\cup_{i}^{\prime}$ in the sense of Definition $\left(^{*}\right)$.

Define $\theta_{i}^{\prime}: C^{\prime} \rightarrow C^{\prime}$ by $\theta_{i}^{\prime}(x)=x \bigcup_{i}^{\bigcup^{\prime}} x+x \bigcup_{i+1}^{\prime} \delta^{\prime} x$, then by Proposition 1, $\theta_{1}^{\prime}$ induces Steenrod operations ${ }_{B}^{i} S T_{i},{ }_{F} S T_{i}^{i+1}$ in the spectral sequence associated with $G(\Gamma \otimes \Gamma, \Lambda \otimes \Lambda)$ such that

$$
{ }_{B} S T_{i}: E_{r}^{p, q}(\Gamma \otimes \Gamma) \longrightarrow E_{2 \gamma-2}^{2 p-i, 2 q}(\Gamma \otimes \Gamma) \text { for } \quad \infty \geqq \gamma \geqq 2
$$

and

$$
{ }_{F} S T_{i}: E_{\gamma}^{p, q}(\Gamma \otimes \Gamma) \longrightarrow E_{\gamma}^{p, 2 q+p-i}(\Gamma \otimes \Gamma) \text { for } \quad \infty \geqq \gamma \geqq 1
$$


They are all $Z_{2}$-homomorphisms.

Proposition 8. The following diagram is commutative

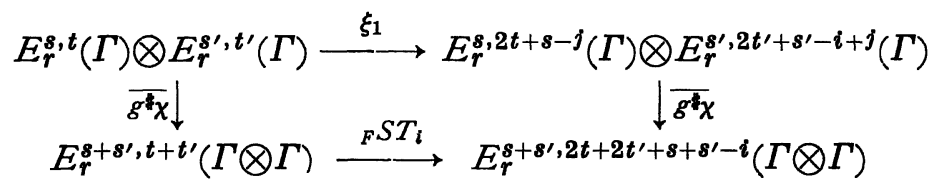

where $\xi_{1}=\sum_{j=0}^{i} F S T_{j} \otimes_{F} S t_{i-j}$ for $1 \leqq r \leqq \infty$. Similarly ${ }_{B} S T_{i}$ with $2 \leqq r \leqq \infty$.

Proof. Let $x=\bar{u} \in E_{r}^{s, t}(\Gamma)$ and $y=\bar{v} \in E_{r}^{s^{\prime}, t^{\prime}}(\Gamma)$, then,

$$
\begin{gathered}
S T_{i} \overline{g^{\sharp} \chi}(x \otimes y)=S T_{i} \overline{\left(g^{\sharp} \chi(u \otimes v)\right)} \\
=\overline{\theta_{i}^{\prime} g^{\sharp}(u \otimes v)}=\overline{g^{\sharp}(u \otimes v) \bigcup_{i}^{\prime} g^{\sharp}(u \otimes v)+g^{\sharp}(u \otimes v) \underset{i+1}{\bigcup} \delta g^{\sharp}(u \otimes v) .}
\end{gathered}
$$

Let $S T_{i} \overline{g^{*}}(x \otimes y)$ be represented by

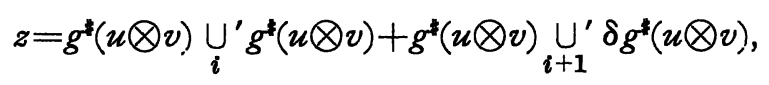

then

$$
\begin{aligned}
& z=g^{*}\left(\sum_{j=0}^{i} h^{j *} \otimes h^{i-j *}\right)\left(t^{*} \otimes 1 \otimes 1\right)^{i}\left(1 \otimes t^{*} \otimes 1\right)\left(f^{*} \otimes f^{*}\right)\left(g^{\star} \otimes g^{\star}\right) \\
& (u \otimes v \otimes u \otimes v)+g^{*}\left(\sum_{\alpha=0}^{i+1} h^{\alpha \star} \otimes h^{i+1-\alpha}\right)\left(t^{*} \otimes 1 \otimes 1\right)^{i+1} \\
& \left.\left(1 \otimes t^{*} \otimes 1\right)\left(f^{*} \otimes f^{*}\right)\left(g^{*} \otimes g^{*}\right)(u \otimes v \otimes \delta u \otimes v)+u \otimes v \otimes u \otimes \delta v\right) \\
& =g^{*}\left(\sum_{j=0}^{i} h^{j *}(u \otimes u) \otimes h^{i-j *}(v \otimes v)\right)+g^{*}\left(\sum_{\alpha=0}^{i+1} h^{\alpha *}(u \otimes u) \otimes h^{i+1-\alpha *}(v \otimes \delta v)\right) \\
& +g^{\star}\left(\sum_{\alpha=0}^{i+1} h^{\alpha \star} \otimes h^{i+1-\alpha \ddagger}\right)\left(t^{\ddagger} \otimes 1 \otimes 1\right)^{i+1}(u \otimes \delta u \otimes v \otimes v)
\end{aligned}
$$

Case 1. $i$ is odd, then

$$
\begin{aligned}
& z=g^{*}\left(\sum_{j=0}^{i} h^{j *}(u \otimes u) \otimes h^{i-j *}(v \otimes v)\right. \\
& \left.+\sum_{\alpha=0}^{i} h^{\alpha \star}(u \otimes u) \otimes h^{i+1-\alpha *}(v \otimes \delta v)\right) \\
& +g^{*}\left(\sum_{\alpha=1}^{i+1} h^{\alpha \ddagger}(u \otimes \delta u) \otimes h^{i+1-\alpha *}(v \otimes v)\right) \\
& +g^{*}\left(h^{i+1 *}(u \otimes u) \otimes h^{o \ddagger}(v \otimes \delta v)\right) \\
& +g^{*}\left(h^{* *}(u \otimes \delta u) \otimes h^{i+1 *}(v \otimes v)\right) \\
& =g^{\sharp}\left\{\sum _ { j = 0 } ^ { i } ( h ^ { j \sharp } ( u \otimes u ) + h ^ { j + 1 } ( u \otimes \delta u ) ) \otimes \left(h^{i-j \sharp}(v \otimes v)\right.\right.
\end{aligned}
$$




$$
\begin{aligned}
& \left.\left.+h^{i-j+1 *}(v \otimes \delta v)\right)\right\}+g^{*}\left(\sum_{j=0}^{i} h^{j+1 *}(u \otimes \delta u)\right. \\
& \left.\quad \otimes h^{i-j+1 *}(v \otimes \delta v)\right)+g^{\ddagger}\left(h^{i+1 *}(u \otimes u) \otimes h^{o \sharp}(v \otimes \delta v)\right) \\
& +g^{*}\left(h^{o \sharp}(u \otimes \delta u) \otimes h^{i+1 *}(v \otimes v)\right) .
\end{aligned}
$$

On the other hand,

$$
\overline{g^{\ddagger} \chi} \sum_{j=0}^{i}\left(S t_{j} \otimes S t_{i-j}\right)(x \otimes y)
$$

can be represented by

$$
\begin{aligned}
z^{\prime}= & g^{*} \sum_{j=0}^{i} \theta_{j}(u) \otimes \theta_{i-j}(v) \\
= & g^{*}\left\{\sum _ { j = 0 } ^ { i } ( h ^ { j \sharp } ( u \otimes u ) + h ^ { j + 1 * } ( u \otimes \delta u ) ) \otimes \left(h^{i-j *}(v \otimes v)\right.\right. \\
& \left.\left.+h^{i-j+1 *}(v \otimes \delta v)\right)\right\}
\end{aligned}
$$

Now, we only have to show that $\bar{w}=0$, where

$$
\begin{gathered}
w=g^{\ddagger}\left(\sum_{j=0}^{i} h^{j+1 *}(u \otimes \delta u) \otimes h^{i-j+1 *}(v \otimes \delta v)\right)+g^{\ddagger}\left(h^{o \ddagger}(u \otimes \delta u)\right. \\
\otimes h^{i+1 *}(v \otimes v)+h^{i+1 *}(u \otimes u) \otimes h^{o \ddagger}(v \otimes \delta v) .
\end{gathered}
$$

But

$$
\begin{aligned}
w^{\prime}=u \cup \delta u \otimes v & \bigcup_{i-j+1} \delta v+u \bigcup_{i+1} u \otimes v \bigcup_{o} \delta v+ \\
& u \bigcup_{o} \delta u \otimes v \bigcup_{i+1} v \\
& \in F^{\delta+\delta^{\prime}+1} \cap F^{2 s+2 s^{\prime}-i+1}
\end{aligned}
$$

and

$$
\begin{aligned}
& \delta^{\prime} w^{\prime}=\left(u \bigcup_{j} \delta u+\delta u \bigcup_{j} u+\delta u \bigcup_{j+1} \delta u\right) \otimes\left(v \bigcup_{i-j+1} \delta v\right) \\
& +\left(u \cup_{j+1} \delta u\right) \otimes\left(v \cup_{i-j} \delta v+\delta v \cup_{i-j} v+\delta v \cup_{i-j+1} \delta v\right) \\
& +\left(\delta u \bigcup_{i+1} u+u \cup \delta u\right) \otimes(v \cup \delta v)+(u \cup u) \otimes(\delta v \cup \delta v) \\
& +(\delta u \cup \delta u) \otimes(v \cup v)+(u \cup \delta u) \otimes(\delta v \cup v+v \cup \delta v) \text {. } \\
& \in F^{s+s^{\prime}+r} C \cap F^{2 s+88^{\prime}+2 r-i-1} C \subset F^{s+8^{\prime}+r} C \cap F^{2 s+2 s^{\prime}+2 r-i-2} C \\
& \Rightarrow w \in Z_{r-1}^{s+s^{\prime}+1,2\left(t+t^{\prime}\right)+s+s^{\prime}-i-1}(\Gamma \otimes \Gamma) \\
& \cap Z_{2 r-3}^{2\left(s+s^{\prime}\right)-i+1,2\left(t+t^{\prime}\right)-1}(\Gamma \otimes \Gamma) \Rightarrow \bar{w}=0 .
\end{aligned}
$$

Case 2. $i$ is even, then

$$
\begin{aligned}
z= & g^{*}\left(\sum_{j=0}^{i} h^{j \neq}(u \otimes u) \otimes h^{i-j *}(v \otimes v)\right) \\
& +g^{*}\left(\sum_{\alpha=0}^{i-1} h^{\alpha \star}(u \otimes u) \otimes h^{i+1-\alpha \star}(v \otimes \delta v)\right)
\end{aligned}
$$




$$
\begin{aligned}
& +g^{*}\left(\sum_{\alpha=0}^{i-1}\left(h^{\alpha \ddagger}(\delta u \otimes u) \otimes h^{i+1-\alpha \star}(v \otimes v)\right)\right) \\
& =g^{*} \sum_{j=0}^{i}\left\{( h ^ { j k } ( u \otimes u ) + h ^ { j - 1 * } ( \delta u \otimes u ) ) \otimes \left(h^{i-j *}(v \otimes v)\right.\right. \\
& \left.\left.+h^{i-j+1 *}(v \otimes \delta v)\right)\right\}+g^{*}\left(\sum_{j=0}^{i} h^{j+1}(\delta u \otimes u) \otimes\right. \\
& \left.h^{i-j+1 *}(v \otimes \delta u)\right)+g^{\sharp}\left(h^{i+1}(u \otimes u) \otimes h^{o \ddagger}(v \otimes \delta v)\right) \\
& +g^{\sharp}\left(h^{o \ddagger}(\delta u \otimes u) \otimes h^{i+1}(v \otimes v)\right) \text {. }
\end{aligned}
$$

Now Lemma 1 says that $\overline{u \bigcup_{j} u+u \bigcup_{j+1} \delta u}=\overline{u \bigcup_{j} u+\delta u \bigcup_{j+1} u}$, and the rest of the proof in this case will be the same as in Case 1. Hence the proof is completed.

Consider the following diagram

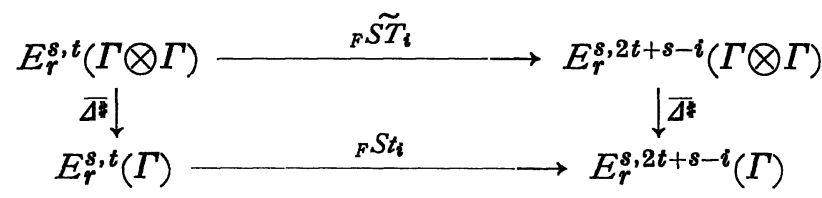

where ${ }_{F} \widetilde{S T}$ induced by $\tilde{H}^{i}: B(\Gamma \otimes \Gamma) \rightarrow B(\Gamma \otimes \Gamma)^{2}$ and $\tilde{H}^{i}$ is defined in the same way $h^{i}: B(\Gamma) \rightarrow B(\Gamma)^{2}$ was defined previously. The above diagram is commutative (Proposition 6).

Now if $\tilde{H}^{i}$ and $H^{i}=(f \otimes f)(1 \otimes t \otimes 1)(t \otimes 1 \otimes 1)^{i}\left(\sum_{j=0}^{i} h^{j} \otimes h^{i-j}\right) g$ satisfy Proposition 7, then

$$
{ }_{F} \widetilde{S T_{i}}={ }_{F} S T_{i} \text { and }{ }_{B} \widetilde{S T_{i}}={ }_{B} S T_{i}
$$

From [6], there exist homotopies $E_{i}: \tilde{H}_{i} \rightarrow H_{i}$. Now, if a family of homotopies $E_{i}$ can be obtained satisfying the filtration conditions of Proposition 7, then we have the following (Cartan formula). For $\xi_{1}, \xi_{2}$ $\in E_{r}(\Gamma)$.

$\begin{aligned} \text { 1) }{ }_{F} S t_{i}\left(\xi_{1} \cdot \xi_{2}\right) & =\sum_{j=0}^{i}{ }_{F} S t_{j} \xi_{1}{ }_{F} S t_{i-j} \xi_{2} \\ \text { 2) }{ }_{B} S t_{i}\left(\xi_{1} \cdot \xi_{2}\right) & =\sum_{j=0}^{i}{ }_{B} S t_{j} \xi_{1}{ }_{B} S t_{i-j} \xi_{2}\end{aligned}$

where $\xi_{1} \cdot \xi_{2}=\overline{\Delta^{*} g^{*} \chi}\left(\xi_{1} \otimes \xi_{2}\right)$.

\section{References}

[1] Adams, J. F., On the non-existence of elements of Hopf invariant one, Ann. Math. 72 (1960), 20-104. 
[2] Araki, S., Steenrod reduced powers in the spectral sequence associated with a fibering, Mem. Fac. Sci. Kyushu Univ. Ser. A 11 (1957), 15-64, 81-97.

[ 3 ] Gugenheim, V. K. A. M., On twisted tensor products of algebras, (mimeographed), John Hopkins Univ., Baltimore, Md., 1960.

[ 4 ] Uehara, H., On Cohomology of Hopf Algebras, Lecture notes, Universitat des Saarlandes, 1970.

[5] - Algebraic Steenrod operations in the spectral sequence associated with a pair of Hopf algebras, Osaka J. Math., 9 (1972), 131-141.

[6] Uehara, H., Al-Hashimi, B., Brenneman, F.S. and Herz, G., On higher homotopies, (to appear in Manuscripta Mathematica).

[7] Vazquez, R., Note on Steenrod squares in the spectral sequence of a fibre space, Bol. Sor. Math. Mexican, 2 (1957), 1-8.

[8] Zachariou, A., Higher Alexander-Whitney maps and cup-i-products, Matematisk Institut, Aarhus Univ, preprint series, 1969/70, No. 6. 